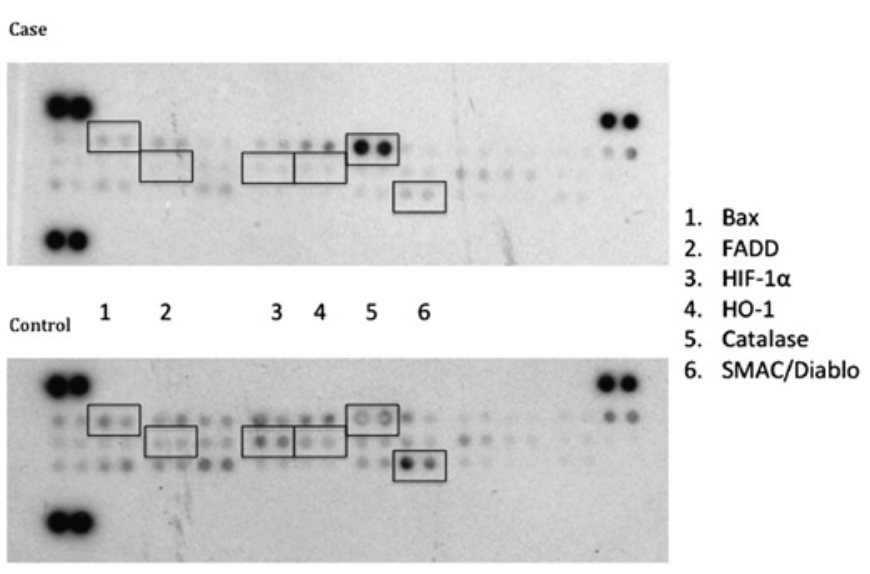

Abstract P02 Figure 3 Examples of apoptosis array for case and control.

$(p<0.03)$. Systemic arterial M30 levels were intermediate between the PV and HV levels (Abstract P02 figure 1). Analysis of apoptosis arrays showed significant downregulation of a number of apoptosisassociated proteins, including Bax, HIF-1, FADD and SMAC/Diablo $(p<0.04)$ (Abstract P02 figure 2). Catalase was markedly elevated compared to controls in three of the four AALF patients. Histological evaluation revealed confluent hepatocellular loss, epithelial regenerative activity and an absence of apoptotic bodies.

Conclusion In this large cohort of PALF patients we have demonstrated the presence of elevated M30 levels and a correlation between caspase three activation and poor clinical outcome. The transhepatic M30 gradient, down regulation of apoptosis-associated proteins and histological appearances indicate that hepatocellular apoptosis might not be the major source of circulating M30. Our data also indicate that in established PALF, apoptosis in non-hepatic epithelial tissues may predominate and is likely to reflect incipient multi-organ failure, with resulting poor outcomes.

\section{P03 THE DIAGNOSTIC VALUE OF TRANSIENT ELASTOGRAPHY COMPARED TO CLINICAL ACUMEN, LABORATORY TESTS AND ULTRASOUND? IS THERE ADDED VALUE?}

doi:10.1136/gutjnl-2011-300857a.3

${ }^{1} \mathrm{G}$ E Dolman, ${ }^{2} \mathrm{~A}$ Ferguson, ${ }^{3} \mathrm{~S}$ Harris, ${ }^{4} \mathrm{~A} M$ Zaitoun, ${ }^{2} \mathrm{M}$ James, ${ }^{2} \mathrm{~S} \mathrm{D}$ Ryder, ${ }^{2} \mathrm{G} P$ Aithal, ${ }^{2} \mathrm{I} N \mathrm{~N}$ Guha. ${ }^{1}$ Nottingham Digestive Diseases Centre/Biomedical Research Unit; ${ }^{2}$ Nottingham Digestive Diseases Centre/Biomedical Research Unit, University of Nottingham, Nottingham, UK; ${ }^{3}$ Public Health Sciences and Medical Statistics, University of Southampton, UK; ${ }^{4}$ Department of Histopathology, Nottingham University Hospitals NHS Trust

Introduction The diagnostic performance of transient elastography (TE), and other non-invasive biomarkers of fibrosis, is assessed by direct comparison with liver biopsy. However clinical acumen laboratory tests and ultrasonography are utilised for the assessment of fibrosis in clinical practice.

Aim The aim of this study was to assess the incremental value of elastography compared to routine diagnostic tools.

Method We included consecutive patients with both fibroscan and biopsy data. Patients with decompensated cirrhosis or suboptimal fibroscan readings were excluded (success rate $<60 \%$ or IOR/median $>0.21$ ). Four consultant/attending hepatologists (who were blinded to TE and biopsy results) were asked to assess the severity of fibrosis on the basis of anonymised clinical data. Simple laboratory tests (eg, full blood count, liver function tests and clotting) and ultrasonography for each case were then given to the clinicians to assess the incremental increase in diagnostic performance. One independent pathologist formally assessed the degree of fibrosis on biopsy, which was the reference standard. Receiver Operating Characteristics (ROC) curves were calculated for (1) clinical acumen (2) clinical acumen + laboratory tests + ultrasonography and (3) $\mathrm{TE}$, for the prediction of significant fibrosis (greater or equal to F2) and cirrhosis.

Results 130 patients were enrolled in the study with paired data and a biopsy deemed adequate for staging. The cohort (65\% male; mean age 46 years) was of mixed aetiology ( $15 \%$ ALD, $48 \%$ chronic viral hepatitis, $24 \%$ NAFLD, $24 \%$ other). The average biopsy length was $23 \mathrm{~mm}$ with 16 portal tracts. The median TE reading was 6.3 (median IOR 0.8 and $100 \%$ success rate).

Conclusion There appears to be little additional benefit in AUC performance of transient elastography to diagnose cirrhosis compared to clinical acumen and routinely available tests. There is however incremental diagnostic benefit for the assessment of significant fibrosis. The baseline performance of simple diagnostic tools, which will vary depending on the stage of fibrosis, needs to be accounted for when assessing liver biomarker performance.

\section{P04 A DOSE EFFECT OF THE DISEASE RISK GENE HLA DR3 CONTRIBUTES TO NUMERICAL AND FUNCTIONAL IMPAIRMENT OF CD4+CD25+ REGULATORY T CELLS IN PATIENTS WITH AUTOIMMUNE HEPATITIS}

doi:10.1136/gutjnl-2011-300857a.4

P Wang, J Underhill, M S Longhi, G Mieli-Vergani, Y Ma, D Vergani. Institute of Liver Studies, King's College London School of Medicine, King's College Hospital, Denmark Hill, London, UK

Introduction Autoimmune diseases are frequent among first degree relatives (FDR) of patients with autoimmune hepatitis (AIH), but concordance for $\mathrm{AIH}$ is rare. A numerical and functional impairment of $\mathrm{CD} 4{ }^{\text {pos }} \mathrm{CD} 25^{\text {pos }}$ regulatory $\mathrm{T}$ cells (Tregs) is described in $\mathrm{AIH}$ patients, but no study has addressed Treg status in their FDR.

Aim To define whether the defect of Tregs in AIH is inherited and is associated with disease predisposing HLA genes.

Abstract P03 Table 1 AUC performance for the assessment of significant fibrosis and cirrhosis

\begin{tabular}{lllllc}
\hline & Clinician & $\begin{array}{l}\text { Clinical acumen } \\
\text { AUC }\end{array}$ & $\begin{array}{l}\text { Clinical acumen/lab } \\
\text { tests/radiology AUC }\end{array}$ & $\begin{array}{l}\text { TE alone } \\
\text { AUC }\end{array}$ & $\begin{array}{l}\text { p Value for AUC of } \\
\text { TE vs clinical/lab/ } \\
\text { radiology* }\end{array}$ \\
\hline Detection of & 1 & 0.56 & 0.55 & 0.78 & 0.0003 \\
significant fibrosis & 2 & 0.52 & 0.56 & 0.81 & 0.0001 \\
& 3 & 0.56 & 0.53 & 0.87 & $<0.0001$ \\
& 4 & 0.59 & 0.52 & 0.78 & $<0.0001$ \\
Detection & 1 & 0.63 & 0.70 & 0.87 & 0.0268 \\
of cirrhosis & 2 & 0.65 & 0.73 & 0.82 & 0.6693 \\
& 3 & 0.77 & 0.77 & 0.89 & 0.1367 \\
& 4 & 0.65 & 0.80 & 0.84 & 0.6705 \\
\hline
\end{tabular}


Method 44 children with AIH (33 AIH-1 and 11 AIH-2, median age 13.5 yrs, 23 females), 65 FDR from 34 families [23 fathers, 47 yrs (38-58); 28 mothers, 44 yrs (24-53) and 14 siblings, 7 females, 13 yrs (5-24)] and 42 healthy subjects [HS, 36 yrs (22-54), 37 females] were studied. Tregs were purified from PBMCs using immunomagnetic beads and their phenotype and frequency was assessed by flowcytometry. CD2 $5^{\text {neg }}$ cells were used as responders in co-culture with Tregs and their proliferation was measured by ${ }^{3} \mathrm{H}$ thymidine incorporation. HLA genotyping was performed by PCR using gene specific primers.

Results The frequency of the disease predisposing gene HLA DR3 was significantly higher in patients $(71 \%)$ and their FDR $(56 \%)$ than in HS $(23 \%, p<0.0001$ and $p<0.005)$. The frequency of homozygous DR3 was higher in patients $(29 \%)$ than in FDR $(9 \%, p=0.015)$ and HS $(0 \%, p=0.001)$. In patients the frequency of HLA A1-B8-DR3 haplotype $(42 \%)$ was higher than in FDR $(27 \%, p=0.15)$ and HS $(16 \%, \mathrm{p}=0.02)$. The frequency of conventional CD $4{ }^{\text {pos }} \mathrm{CD} 25^{\mathrm{pos}}$ Tregs was lower in patients $(6.2 \% \pm 0.5)$ than in FDR $(9.3 \% \pm 0.7$, $\mathrm{p}=0.0016)$ and HS $(9.7 \% \pm 0.8, \mathrm{p}=0.001)$. Though the frequency of $\mathrm{CD} 4{ }^{\text {pos }} \mathrm{CD} 25^{\text {high }} \mathrm{CD} 127^{\text {neg }}$ 'True' Tregs was similar among patients, FDR and HS $(6.0 \% \pm 0.6,6.3 \% \pm 0.4$ and $6.2 \% \pm 0.5)$, their suppressive function was lower in patients $\left(13.6 \%\right.$ reduction of CD2 $5^{\text {neg }}$ cell proliferation) than in $\operatorname{FDR}(28.8 \%, \mathrm{p}=0.007)$ and $\mathrm{HS}(36.9 \%$, $\mathrm{p}<0.0001)$. Among subjects positive for HLA DR3, the frequency of conventional Tregs was lower in patients $(5.6 \% \pm 0.5)$ than in FDR (8.4\% $\pm 0.97, p=0.01)$ and in HS $(9.1 \% \pm 1.4, p=0.006)$. Among subjects positive for HLA A1-B8-DR3 haplotype, the frequency of Tregs was lower in patients $(6.0 \% \pm 0.7)$ than in FDR $(9.1 \% \pm 1.5$, $\mathrm{p}=0.045)$ and HS $(9.8 \% \pm 1.8, \mathrm{p}=0.02)$.

Conclusion A numerical and functional impairment of Tregs in $\mathrm{AIH}$ patients is associated with possession of HLA disease predisposing genes, and in particular HLA DR3 homozygosity. Possession of DR3 was not associated to a similar immune regulatory impairment in FDR and HS, suggesting that a gene dose effect contributes to the impairment of immunoregulation and to the development of AIH.

\section{P05 COMBINATION OF SERUM BIOMARKERS FOR THE DIAGNOSIS OF HEPATOCELLULAR CARCINOMA}

doi:10.1136/gutjnl-2011-300857a.5

${ }^{1} \mathrm{P}$ Johnson, ${ }^{1} \mathrm{M}$ Teng, ${ }^{1} \mathrm{~S}$ Pirrie, ${ }^{2} \mathrm{D}$ Palmer, ${ }^{1} \mathrm{~S}$ Berhane, ${ }^{1} \mathrm{D}$ Stocken. ${ }^{1}$ University of Birmingham, UK; ${ }^{2}$ University of Liverpool, UK

Introduction The most commonly used serum biomarker for the diagnosis of hepatocellular carcinoma (HCC) is $\alpha$ fetoprotein (AFP). However, AFP is also modestly raised in patients with chronic liver disease, particularly in those who are at a higher risk of developing HCC. A number of other circulating markers such as des- $\gamma$ carboxy prothrombin (DCP) and the Lens culinaris agglutinin-reactive fraction of $\alpha$-fetoprotein (L3) have been shown to have diagnostic discrimination which may, in certain situations, be superior to AFP. Aim We therefore aimed to build a model that combined these biomarkers with baseline liver function tests, in order to identify those factors which predict HCC and might improve diagnostic efficacy of AFP alone.

Method A prospective study was specifically designed to assess the diagnostic efficacy of biomarkers. 461 serum samples were collected between 2007 and 2011: 252 were controls samples from patients with chronic liver disease with no evidence of HCC within 6 months of the sample; 59 were from patients with early HCC which was potentially curable and 150 were from patients with late HCC. AFP, AFP-L3 and DCP were measured in serum samples using a new micro total analysis system (Wako Chemicals GmbH, Neuss, Germany). Patients were classified into two groups (HCC, control) for the primary analysis. Independent predictors of HCC were identified from multivariable logistic regression analyses. The impact of AFP, DCP and L3 are reported based on the area under the receiver operator curve (AUC).

Results Univariate analysis showed AFP alone to have $\mathrm{OR}=2.96$ (2.34 to $3.7395 \% \mathrm{CI}$ ), AUC $=0.8723, \mathrm{p}<0.001$. A multivariable model selected age, sex, AST, ALP and INR as independent predictors of HCC with an overall model AUC $=0.8857$. Including AFP, DCP and L3 to this model increased the AUC to 0.9489, 0.9290, 0.9254 respectively. The final model included age, sex, AFP, DCP and INR gave an AUC of 0.9661 (95\% CI 0.95 to 0.98 ) for all patients and 0.944 (95\% CI 0.914 to 0.974 ) for early HCC patients.

Conclusion A combination of serum markers may produce a clinically useful diagnostic test for HCC compared to AFP alone. Further validation of our model is underway on an independent dataset. These findings suggest that our model may enhance or replace the current screening approach for patients with liver cirrhosis, particularly since it appears to have efficacy even in patients with early, potentially curable, disease.

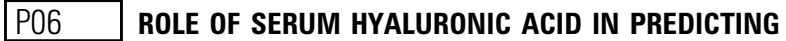 MORTALITY IN PATIENTS WITH CHRONIC LIVER DISEASE}

doi:10.1136/gutjnl-2011-300857a.6

${ }^{1} \mathrm{~N}$ Plevris, ${ }^{2} \mathrm{~N}$ C Mcavoy, ${ }^{2} \mathrm{P}$ C Hayes. ' University of Edinburgh, UK; ${ }^{2}$ Department of Hepatology, Royal Infirmary of Edinburgh, UK

Introduction Currently liver biopsies are used to assess extent of liver fibrosis and provide prognostic information in liver disease. Hyaluronic acid (HA) is a non-invasive serum marker that has been shown to correlate well with different degrees of hepatic fibrosis. Despite this the prognostic abilities of HA still remain to be established.

Aim In this study we aim to establish the relationship between levels of serum HA and survival, as well as determine the sensitivity and specificity of HA for predicting death in patients with chronic liver disease of varying aetiology.

Method Patients seen at the department of hepatology, Royal Infirmary of Edinburgh between 1995 and 2010 who had serum HA levels measured were followed up for death from the date of HA measurement until the 1 November 2010 by examination of clinical records on TRAK (NHS Lothian). The cumulative probability for survival from liver related death and overall deaths at 1-, 5- and 8-year follow-up was determined. Receiver operating characteristic (ROC) curves were generated to assess the sensitivity and specificity of HA levels at predicting liver-related death at 1, 3 and 5 years.

Results HA levels were available for 632 patients. The median follow-up time (from HA measurement to death or 'last known alive') was 2.7 years (range $0.0-8.0$; IOR 0.9-4.4). Survival analysis showed that HA levels $>400 \mu \mathrm{g} / \mathrm{l}$ are associated with a significantly lower probability of survival from liver related death compared to patients with values of $<100 \mu \mathrm{g} / 1$ and $100-400 \mu \mathrm{g} / \mathrm{l}$ at 1 year $(88 \%$, $\mathrm{p}<0.01), 5$ years $(68 \%, \mathrm{p}<0.001)$ and 8 years $(49 \%, \mathrm{p}<0.05)$. In addition, the probability of survival from liver related death at 8 years was also significantly less in patients with HA value of $100-400 \mu \mathrm{g} / \mathrm{l}$ compared to patients with HA $<100 \mu \mathrm{g} / 1$ (83\%, $p<0.05)$. Similar results were observed when assessing probability of survival from overall deaths. The unadjusted area under the ROC curve for predicting liver related mortality for HA at 1-, 3- and 5 years was 0.89 (CI 95\% 0.84 to 0.94 ), 0.85 (CI 95\% 0.79 to 0.91 ) and 0.79 (CI $95 \% 0.71$ to 0.87 ) respectively. Optimum cut-off value for 1-, 3-and 5 year for predicting liver death was $326 \mu \mathrm{g} / 1$ (88\% sensitivity/79\% specificity), $267 \mu \mathrm{g} / 1$ (74\% sensitivity/79\% specificity) and $203 \mu \mathrm{g} / 1$ (71\% sensitivity/72\% specificity) respectively.

Conclusion In this study we have shown that patients with high levels of serum HA have an increased liver and all cause mortality. In addition, HA levels are both sensitive as well as specific at determining liver related mortality. This highlights the potential use of 